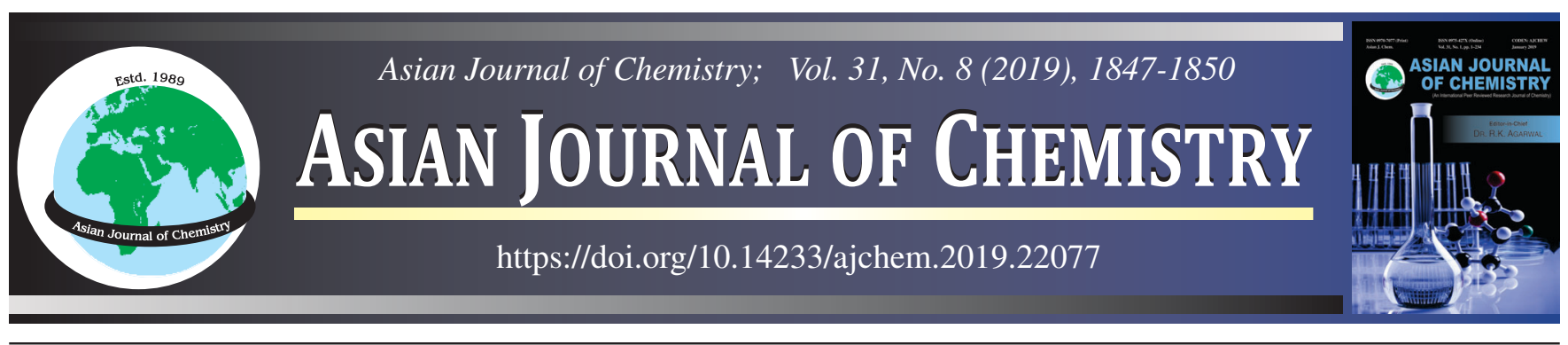

\title{
Chalcone Ditosylates as Potent Precursor for Synthesis of Some 4,5-Disubstituted Isoxazoles with Antioxidant and Anti-inflammatory Activities
}

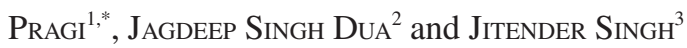

${ }^{1}$ Department of Pharmaceutical Sciences, Indra Kumar Gujral Punjab Technical University, Kapurthala-144603, India

${ }^{2}$ Shivalik College of Pharmacy, Nangal-140124, India

${ }^{3}$ Lord Shiva College of Pharmacy, Sirsa-125055, India

*Corresponding author: E-mail: arorapragi@gmail.com

Received: 14 March 2019;

Accepted: 26 April 2019;

Published online: 28 June 2019;

AJC-19464

The present work emphasizes on the synthesis of a series of 4,5-disubstituted isoxazole derivatives of $\alpha, \beta$-chalcone ditosylates which were synthesized by the reaction of $\alpha, \beta$-chalcone ditosylates with hydroxylamine hydrochloride. Various $\alpha, \beta$-chalcone ditosylates were prepared by the reaction of respective chalcones with hydroxyl (tosyloxy)iodobenzene.The synthesized compounds were characterized and subsequently evaluated for anti-inflammatory and antioxidant properties.

Keywords: $\alpha, \beta$-Chalcone ditosylates, 4,5-Disubstituted isoxazoles, hydroxyl(tosyloxy)iodobenzene, Microbial activity.

\section{INTRODUCTION}

Isoxazoles are an important class of heterocyclic compounds and their chemical properties have been studied over the years. Isoxazole derivatives have been useful as a versatile building block for the synthesis of organic compounds. Isoxazoles can be converted into various important synthetic units like $\beta$ hydroxy ketones [1-7], $\gamma$-amino alcohols [8,9], $\alpha, \beta$-unsaturated oxime $[10,11]$ and $\beta$-hydroxy nitriles $[12,13]$. Along with these, isoxazole derivatives have been used in synthetic investigation as of their known biological activities and pharmacological properties like hypoglycemic effect [14], analgesic [15], anti-inflammatory [16], antibacterial activities [17] and anticancer activity [18]. 4,5-Diarylisoxazole derivatives also act as potent therapeutic agents for the treatment of cancer [19] inhibition of cyclooxygenase- 2 and have analgesic and anti-inflammatory activities [20]. These compounds are also helpful for the treatment of angiogenesis-related diseases including cancers, rheumatoid arthritis and psoriasis [21].

In view of the above, the present study focuses on the synthesis of 4,5-disubstituted isoxazole derivatives from $\alpha, \beta$ chalcone ditosylates. A new and convenient route to synthesis of 4,5-diarylisoxazoles from chalcone ditosylates had also been reported [22]. $\alpha, \beta$-Chalcone ditosylates reacts with hydroxyl- amine hydrochloride to produce 1,2-aryl shift and thus give a novel method for the synthesis of 4,5-disubstituted isoxazoles. Different chalcone ditosylates were prepared by the reaction of chalcones with Koser's reagent (hydroxyl(tosyloxy)iodobenzene, HTIB) as described by Rebrovic and Koser [23], where they reported a stereo-specific syn-1,2-ditosyloxylation of carbon-carbon double bond using HTIB.

\section{EXPERIMENTAL}

Melting points of the synthesized compounds were determined by using open capillary method and are uncorrected. TLC was performed to check the purity and to monitor the reaction. The IR spectra were recorded on Shimadzu FTIR 8400 spectrophotometer using $\mathrm{KBr}$ pallets. ${ }^{1} \mathrm{H}$ NMR spectra were obtained in $\mathrm{CDCl}_{3}$ on a Bruker Spectrometer at $400 \mathrm{~Hz}$. The chemical shifts are reported in ppm $(\delta)$ in relation to tetramethylsilane (TMS) as an internal standard. Coupling constants $(J)$ are expressed in Hertz $(\mathrm{Hz})$. All the chemicals and reagents used were purchased from Merck and Sigma Aldrich.

Ethics statements: The protocol was approved by the Institutional Animals Ethics Committee of the M.M. College of Pharmacy (MMCP-IAEC-6 dated: 04/08/2018). All the pharmacological activities were carried out as per CPCSEA

This is an open access journal, and articles are distributed under the terms of the Attribution 4.0 International (CC BY 4.0) License. This license lets others distribute, remix, tweak, and build upon your work, even commercially, as long as they credit the author for the original creation. You must give appropriate credit, provide a link to the license, and indicate if changes were made. 
(Committee for the purpose of control and supervision of experiments on animals) norms.

General procedure for the synthesis of 4,5-disubstituted isoxazoles from $\alpha, \beta$-chalcone ditosylates: A mixture of chalcone ditosylate (1a; $0.550 \mathrm{~g}, 0.001 \mathrm{~mol}$ ), hydroxylamine hydrochloride $(0.138 \mathrm{~g}, 0.002 \mathrm{~mol})$ and sodium acetate $(0.164 \mathrm{~g}$, $0.002 \mathrm{~mol})$ in ethanol $(25 \mathrm{~mL})$ was refluxed for approximately $3 \mathrm{~h}$. The reaction mixture was poured on the ice-cold water. The resulting mixture was then extracted with dichloromethane $(3 \times 50 \mathrm{~mL})$ and the organic extract obtained was dried over anhydrous sodium sulphate and filtered. Evaporation of dichloromethane in vacuo and the crude product obtained was purified by column chromatography on silica gel (100-200 mesh) using petroleum ether-ethylacetate as eluent to give pure isoxazole.

5-(Naphthalene-3-yl)-4-phenylisoxazole (V1D1I1): Yield: $65 \%$, m.p.: $134-136^{\circ} \mathrm{C}$. IR $\left(v_{\max }, \mathrm{KBr}, \mathrm{cm}^{-1}\right)$ : Peak absent in CO region; ${ }^{1} \mathrm{H} \mathrm{NMR}\left(\mathrm{CDCl}_{3}, 300 \mathrm{MHz}\right) \delta \mathrm{ppm}: 7.32(\mathrm{~m}, 5 \mathrm{H}, \mathrm{ArH})$; 8.29 (s, 1H, C3-isoxazole proton); 6.60-6.98 (m, 7H, ArH). Elemental analysis: calcd. (found) for $\mathrm{C}_{19} \mathrm{H}_{13} \mathrm{NO}$ : C 84.11 (84.10), H 4.83 (4.84).

5-(Naphthalene-3-yl)-4-(4-chlorophenyl)isoxazole (V2D2I2): Yield: $69 \%$, m.p.: $117-119^{\circ} \mathrm{C}$. IR $\left(v_{\max }, \mathrm{KBr}, \mathrm{cm}^{-1}\right)$ : Peak absent in $\mathrm{CO}$ region; ${ }^{1} \mathrm{H}$ NMR $\left(\mathrm{CDCl}_{3}, 300 \mathrm{MHz}\right) \delta \mathrm{ppm}$ : $7.03(\mathrm{~d}, 2 \mathrm{H}, \mathrm{ArH}, J=8.1 \mathrm{~Hz}) ; 7.23$ (d, 2H, ArH, $J=8.1 \mathrm{~Hz})$; 7.81 (s, 1H, C3-pyrazole proton); 6.60-6.98 (m, 7H, ArH). Elemental analysis: calcd. (found) for $\mathrm{C}_{19} \mathrm{H}_{12} \mathrm{NOCl}$ : C 74.64 (74.62), H 3.96 (H 3.97).

5-(Naphthalene-3-yl)-4-(4-nitrophenyl)isoxazole (V3D3I3): Yield: $78 \%$, m.p.: $100-102{ }^{\circ} \mathrm{C}$. IR $\left(v_{\max }, \mathrm{KBr}, \mathrm{cm}^{-1}\right)$ : Peak absent in $\mathrm{CO}$ region; ${ }^{1} \mathrm{H}$ NMR $\left(\mathrm{CDCl}_{3}, 300 \mathrm{MHz}\right) \delta \mathrm{ppm}$ : $6.89(\mathrm{~d}, 2 \mathrm{H}, \mathrm{ArH}, J=8.1 \mathrm{~Hz}) ; 7.21(\mathrm{~d}, 2 \mathrm{H}, \mathrm{ArH}, J=8.1 \mathrm{~Hz})$; 6.58-6.81 (m, 7H, ArH); 8.25 (s, 1H, C3-isoxazole proton). Elemental analysis: calcd. (found) for $\mathrm{C}_{19} \mathrm{H}_{12} \mathrm{~N}_{2} \mathrm{O}_{3}: \mathrm{C} 72.15$ (72.13), H 3.82 (3.83).

5-(Naphthalene-3-yl)-4-(4-methoxyphenyl)isoxazole (V4D4I4): Yield: $70 \%$, m.p.: $97-99^{\circ} \mathrm{C}$. IR $\left(v_{\max }, \mathrm{KBr}, \mathrm{cm}^{-1}\right)$ : Peak absent in $\mathrm{CO}$ region; ${ }^{1} \mathrm{H}$ NMR $\left(\mathrm{CDCl}_{3}, 300 \mathrm{MHz}\right) \delta \mathrm{ppm}$ : $6.91(\mathrm{~d}, 2 \mathrm{H}, \mathrm{ArH}, J=8.1 \mathrm{~Hz}) ; 7.26(\mathrm{~d}, 2 \mathrm{H}, \mathrm{ArH}, J=8.1 \mathrm{~Hz})$; 6.60-6.98 (m, 7H, ArH); 8.27 (s, 1H, C3-isoxazole proton); $3.81\left(\mathrm{~s}, 3 \mathrm{H}, \mathrm{OCH}_{3}\right.$ ). Elemental analysis: calcd. (found) for $\mathrm{C}_{20} \mathrm{H}_{15} \mathrm{NO}_{2}$ : C 79.72 (79.71), H 5.02 (5.02).

5-(Naphthalene-3-yl)-4-(4-fluorophenyl)isoxazole (V5D5I5): Yield: $69 \%$, m.p.: $108-110^{\circ} \mathrm{C}$. IR $\left(v_{\max }, \mathrm{KBr}, \mathrm{cm}^{-1}\right)$ : Peak absent in $\mathrm{CO}$ region; ${ }^{1} \mathrm{H}$ NMR $\left(\mathrm{CDCl}_{3}, 300 \mathrm{MHz}\right) \delta \mathrm{ppm}$ : $7.02(\mathrm{~d}, 2 \mathrm{H}, \mathrm{ArH}, J=8.1 \mathrm{~Hz}) ; 7.21$ (d, 2H, ArH, $J=8.1 \mathrm{~Hz})$; 6.58-6.89 (m, 7H, ArH); 8.27 (s, 1H, C3-isoxazole proton). Elemental analysis: calcd. (found) for $\mathrm{C}_{19} \mathrm{H}_{12} \mathrm{NOF}$ : $\mathrm{C} 78.88$ (78.87), H 4.18 (4.18).

5-(Naphthalene-3-yl)-4-p-tolylisoxazole (V6D6I6): Yield: $73 \%$, m.p.: $127-128^{\circ} \mathrm{C}$. IR $\left(v_{\max }, \mathrm{KBr}, \mathrm{cm}^{-1}\right)$ : Peak absent in CO region; ${ }^{1} \mathrm{H}$ NMR $\left(\mathrm{CDCl}_{3}, 300 \mathrm{MHz}\right) \delta \mathrm{ppm}: 7.14(\mathrm{~d}, 2 \mathrm{H}$, ArH, $J=8.1 \mathrm{~Hz}) ; 7.26$ (d, 2H, ArH, $J=8.1 \mathrm{~Hz}) ; 6.60-6.98(\mathrm{~m}$, $7 \mathrm{H}, \mathrm{ArH}$ ); 2.33 (s, 3H, $\mathrm{CH}_{3}$ ); 8.27 (s, 1H, C3-isoxazole proton). Elemental analysis: calcd. (found) for $\mathrm{C}_{20} \mathrm{H}_{15} \mathrm{NO}$ : C 84.19 (84.18), H 5.30 (5.30).

5-(Naphthalene-3-yl)-4-(4-hydroxyphenyl)isoxazole (V7D7I7): Yield: $71 \%$, m.p.: $122-124^{\circ} \mathrm{C}$. IR ( $\left.v_{\max }, \mathrm{KBr}, \mathrm{cm}^{-1}\right)$ : Peak absent in $\mathrm{CO}$ region; ${ }^{1} \mathrm{H}$ NMR $\left(\mathrm{CDCl}_{3}, 300 \mathrm{MHz}\right) \delta \mathrm{ppm}$ : $7.02(\mathrm{~d}, 2 \mathrm{H}, \mathrm{ArH}, J=8.1 \mathrm{~Hz}) ; 7.13(\mathrm{~d}, 2 \mathrm{H}, \mathrm{ArH}, J=8.1 \mathrm{~Hz})$; 6.54-6.87 (m, 7H, ArH); 8.28 (s, 1H, C3-isoxazole proton); $2.35(\mathrm{~s}, 1 \mathrm{H}, \mathrm{OH})$. Elemental analysis: calcd. (found) for $\mathrm{C}_{19} \mathrm{H}_{13} \mathrm{NO}_{2}$; C 79.43 (79.42), H 4.56 (4.55).

in vitro Antioxidant activity: The antioxidant activity of the compounds were determined by using DPPH $(1,1-$ diphenyl-2-picrylhydrazyl) free radical scavenging method. The given test compound was mixed with $95 \%$ methanol to make a final stock solution of concentration $100 \mu \mathrm{g} / \mathrm{mL}$. From the stock solution different solutions of concentration 10, 20, 40, 60 and $100 \mu \mathrm{g} / \mathrm{mL}$ were prepared. Ascorbic acid was used as a standard and various concentrations of ascorbic acid were prepared as of the test compound. Final mixture consisting of $1 \mathrm{~mL}$ of $0.3 \mathrm{mmol}$ DPPH methanol solution was added to 2.5 $\mathrm{mL}$ of sample solution of different concentrations and allowed to react at room temperature. After $15 \mathrm{~min}$ of incubation period at $37^{\circ} \mathrm{C}$, absorbance was calculated at $517 \mathrm{~nm}$. Control reading was also observed without the test compound.

$$
\text { Scavenging }(\%)=\frac{\mathrm{A}_{\text {control }}-\mathrm{A}_{\text {test compound }}}{\mathrm{A}_{\text {control }}} \times 100
$$

in vivo Anti-inflammatory activity: Carrageenan-induced paw edema method was used for the determination of antiinflammatory activities of the compounds. Adult male rats ( $250 \mathrm{~g}$ ) were used to evaluate anti-inflammatory activity. Animals were divided into ten groups. Each group consists of six animals. The different groups of rats were pre-treated with their respective doses. After $1 \mathrm{~h}$, oedema was induced by administration of $0.1 \mathrm{~mL}$ of $1 \%$ carrageenan suspension into sub-plantar region of left hind paw of each rat and then paw volume was measured by using Plethysmometer (Laboratory enterprises, Nasik) at $0,1,2,3,4 \mathrm{~h}$. Mean \pm SEM for treated and control animals was calculated and compared for each time interval and statistically analyzed. The details of the groups are as follows:

$$
\begin{array}{ll}
\text { Group I: } & \begin{array}{l}
\text { Normal control } \\
\text { Group II: }
\end{array} \\
& \begin{array}{l}
\text { Inflammation control group which received } \\
\text { vehicle (0.25 \% carboxymethylcellulose) }
\end{array} \\
\text { Group III: } & \begin{array}{l}
\text { Standard group treated with (standard) } \\
\text { diclofenac sodium (100 mg/kg p.o.) }
\end{array} \\
\text { Group IV: } & \text { Inflammation + V1D1I1 (100 mg/kg p.o.) } \\
\text { Group V: } & \text { Inflammation + V2D2I2 (100 mg/kg p.o.) } \\
\text { Group VI: } & \text { Inflammation + V3D3I3 (100 mg/kg p.o.) } \\
\text { Group VII: } & \text { Inflammation + V4D4I4 (100 mg/kg p.o.) } \\
\text { Group VIII: } & \text { Inflammation + V5D5I5 (100 mg/kg p.o.) } \\
\text { Group IX: } & \text { Inflammation + V6D6I6 (100 mg/kg p.o.) } \\
\text { Group X: } & \text { Inflammation + V7D7I7 (100 mg/kg p.o.) }
\end{array}
$$

Diclofenac sodium $(20 \mathrm{mg} / \mathrm{kg})$ was used as a standard drug. The adult male rats were fasted overnight with access to water. The synthesized compounds were given at the dose of $150 \mathrm{mg} / \mathrm{kg}$ and were administered through oral route. A $1 \%$ saline suspension of carrageenan was prepared. A $0.05 \mathrm{~mL}$ of this suspension was injected into the planter tissue of left hind paw of rat to induce edema. For control, the animals were injected with equal volume of saline. In order to measure the paw volume of rats Plethysmograph was used. 


\section{RESULTS AND DISCUSSION}

Various derivatives of $\alpha, \beta$-chalcone ditosylates were synthesized by the reaction of chalcone with (hydroxyl(tosyloxy)iodo-benzene, HTIB) by using Koser's method. These chalcone ditosylates were treated with hydroxylamine hydrochloride to give different 4,5-disubstituted isoxazoles (Scheme-I). The mechanism of conversion of ditosylates to 4,5-disubstituted isoxazoles involves 1,2-aryl migrations. All the substrates reacted in the same manner and gave good yield in the range 65-78 \%. The compounds were purified by recrystallization from ethanol and dried under vacuum. The synthesized compounds were characterized by IR, ${ }^{1} \mathrm{H}$ NMR and elemental analysis.

The antioxidant activity results are given in Table-1. All the synthesized compounds were found to be less potent than the standard (ascorbic acid). However, the compounds V5D5I5 and V7D7I7 exhibited moderate antioxidant activity in comparison to the standard. Similarly, the anti-inflammatory activity results are given in Table-2. The compoundsV4D4I4 and V7D7I7 showed remarkable anti-inflammatory activity.

\section{Conclusion}

Various 4,5-disubstituted isoxazoles were prepared from the reaction of chalcone ditosylates and hydroxylamine hydrochloride. All the compounds were characterized by IR, NMR and elemental analysis. The IR spectrum of the product showed the absence of a peak at $1680 \mathrm{~cm}^{-1}$ arising from the carbonyl group of chalcone ditosylate. The ${ }^{1} \mathrm{H}$ NMR spectrum showed a sharp singlet at $\delta=8.2 \mathrm{ppm}$, corresponding to a single proton corresponding to that of $\mathrm{C}(3)-\mathrm{H}$ of the isoxazole ring. Antioxidant property was evaluated by using DPPH free radical scavenging activity. The compounds V5D5I5 and V7D7I7 exhibited moderate antioxidant activity as compared with the standard. Synthesized isoxazoles were tested for in vivo anti-inflammatory activity and the compounds V4D4I4 and V7D7I7 showed good activity when compared to the standard.<smiles>[13CH3]/C=C/C(=O)[AlH2]</smiles>

Chalcone ditosylate

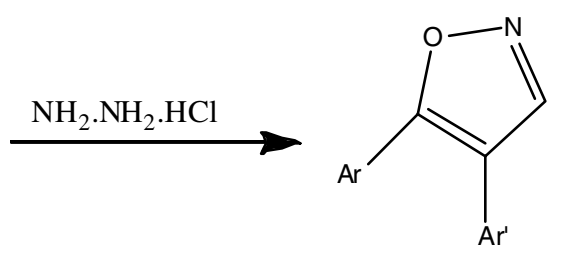

4,5-disubstituted isoxazole

$\begin{array}{llllllll}\text { Compound No. } & \text { V1D1I1 } & \text { V2D2I2 } & \text { V3D3I3 } & \text { V4D4I4 } & \text { V5D5I5 } & \text { V6D6I6 } & \text { V7D7I7 } \\ \mathrm{Ar} & \mathrm{C}_{10} \mathrm{H}_{7} & \mathrm{C}_{10} \mathrm{H}_{7} & \mathrm{C}_{10} \mathrm{H}_{7} & \mathrm{C}_{10} \mathrm{H}_{7} & \mathrm{C}_{10} \mathrm{H}_{7} & \mathrm{C}_{10} \mathrm{H}_{7} & \mathrm{C}_{10} \mathrm{H}_{7} \\ \mathrm{Ar} & \mathrm{C}_{6} \mathrm{H}_{5} & 4-\mathrm{ClC}_{6} \mathrm{H}_{4} & 4-\mathrm{NO}_{2} \mathrm{C}_{6} \mathrm{H}_{4} & 4-\mathrm{MeOC}_{6} \mathrm{H}_{4} & 4-\mathrm{FC}_{6} \mathrm{H}_{4} & 4-\mathrm{MeC}_{6} \mathrm{H}_{4} & 4-\mathrm{OHC}_{6} \mathrm{H}_{4}\end{array}$

Scheme-I: Synthetic sequence of 4,5-disubstituted isoxazoles

TABLE-1

ANTIOXIDANT ACTIVITY OF THE 4,5 DISUBSTITUTED ISOXAZOLES BY USING DPPH FREE RADICAL SCAVENGING METHOD

\begin{tabular}{|c|c|c|c|c|c|c|}
\hline \multirow{2}{*}{ Compound } & \multicolumn{5}{|c|}{ Inhibition $(\%)$} & \multirow{2}{*}{$\begin{array}{l}\text { Antioxidan } \\
\text { IC }_{50} \text { value }\end{array}$} \\
\hline & $10 \mu \mathrm{g} / \mathrm{mL}$ & $20 \mu \mathrm{g} / \mathrm{mL}$ & $40 \mu \mathrm{g} / \mathrm{mL}$ & $60 \mu \mathrm{g} / \mathrm{mL}$ & $100 \mu \mathrm{g} / \mathrm{mL}$ & \\
\hline V1D1I1 & 2.48 & 6.89 & 13.88 & 18.34 & 22.78 & 214.14 \\
\hline V2D2I2 & 1.45 & 7.97 & 12.55 & 16.98 & 20.39 & 240.57 \\
\hline V3D3I3 & 1.76 & 2.09 & 16.80 & 18.65 & 21.09 & 211.03 \\
\hline V4D4I4 & 23.6 & 31.92 & 39.63 & 42.66 & 51.75 & 83.43 \\
\hline V5D5I5 & 25.7 & 37.32 & 44.77 & 52.98 & 63.32 & 59.30 \\
\hline V6D6I6 & 8.73 & 12.12 & 21.57 & 31.09 & 36.98 & 131.70 \\
\hline V7D7I7 & 21.05 & 31.73 & 44.80 & 51.93 & 60.20 & 65.46 \\
\hline Ascorbic acid (Standard) & 48.51 & 60.90 & 69.70 & 80.44 & 92.46 & 1.94 \\
\hline
\end{tabular}

TABLE-2

ANTI-INFLAMMATORY ACTIVITY OF 4,5-DISUBSTITUTED ISOXAZOLE DERIVATIVES

\begin{tabular}{cccccc}
\hline \multirow{2}{*}{ Treatment group } & \multicolumn{3}{c}{ Paw volume $(\mathrm{mm})$ after time $(\mathrm{h})$} \\
\cline { 2 - 6 } & $0 \mathrm{~h}$ & $1 \mathrm{~h}$ & $2 \mathrm{~h}$ & $3 \mathrm{~h}$ & $4 \mathrm{~h}$ \\
\hline V1D1I1 & $0.80 \pm 0.059$ & $1.04 \pm 0.082$ & $1.15 \pm 0.057$ & $1.24 \pm 0.043$ & $1.29 \pm 0.014$ \\
V2D2I2 & $0.85 \pm 0.085$ & $1.13 \pm 0.071$ & $1.22 \pm 0.041$ & $1.30 \pm 0.035$ & $1.36 \pm 0.058$ \\
V3D3I3 & $0.99 \pm 0.038$ & $1.17 \pm 0.077$ & $1.24 \pm 0.037$ & $1.31 \pm 0.015$ & $1.39 \pm 0.039$ \\
V4D4I4 & $0.76 \pm 0.045$ & $0.80 \pm 0.034$ & $0.82 \pm 0.031$ & $0.83 \pm 0.021$ & $0.86 \pm 0.045$ \\
V5D5I5 & $0.91 \pm 0.074$ & $1.10 \pm 0.055$ & $1.20 \pm 0.055$ & $1.29 \pm 0.099$ & $1.31 \pm 0.044$ \\
V6D6I6 & $0.86 \pm 0.022$ & $1.02 \pm 0.037$ & $1.11 \pm 0.050$ & $1.21 \pm 0.066$ & $1.25 \pm 0.030$ \\
V7D7I7 & $0.91 \pm 0.067$ & $1.08 \pm 0.036$ & $1.13 \pm 0.081$ & $1.14 \pm 0.062$ & $1.12 \pm 0.083$ \\
Control & $0.81 \pm 0.026$ & $0.81 \pm 0.025$ & $0.82 \pm 0.017$ & $0.82 \pm 0.019$ & $0.81 \pm 0.023$ \\
Infalmmatory control & $0.97 \pm 0.028$ & $1.15 \pm 0.066$ & $1.25 \pm 0.043$ & $1.30 \pm 0.042$ & $1.32 \pm 0.054$ \\
Diclofenac & $0.88 \pm 0.013$ & $0.96 \pm 0.012$ & $0.83 \pm 0.011$ & $0.82 \pm 0.014$ & $0.78 \pm 0.015$ \\
\hline Vat
\end{tabular}

Values are expressed as mean \pm SEM (standard error mean).

Values are calculated as compared to control using one way ANOVA followed by Dunnet's test. 


\section{CONFLICT OF INTEREST}

The authors declare that there is no conflict of interests regarding the publication of this article.

\section{REFERENCES}

1. A.P. Kozikowski and P.D. Stein, J. Am. Chem. Soc., 104, 4023 (1982); https://doi.org/10.1021/ja00378a049.

2. D.P. Curran, J. Am. Chem. Soc., 105, 5826 (1983); https://doi.org/10.1021/ja00356a021.

3. B.H. Kim, Y.J. Chung and E.J. Ryu, Tetrahedron Lett., 34, 8465 (1993); https://doi.org/10.1016/S0040-4039(00)61360-6.

4. S.H. Andersen, K.K. Sharma and K.B.G. Torssell, Tetrahedron, 39, 2241 (1983);

https://doi.org/10.1016/S0040-4020(01)91946-5.

5. P.G. Baraldi, A. Barco, S. Benetti, S. Manfredini and D. Simoni, Synthesis, 276 (1987);

https://doi.org/10.1055/s-1987-27915.

6. J.W. Bode and E.M. Carreira, Org. Lett., 3, 1587 (2001); https://doi.org/10.1021/o1015885d.

7. A.P. Kozikowski and M. Adamczyk, Tetrahedron Lett., 23, 3123 (1982); https://doi.org/10.1016/S0040-4039(00)88575-5.

8. A.P. Kozikowski and Y.Y. Chen, J. Org. Chem., 46, 5248 (1981); https://doi.org/10.1021/jo00338a049.

9. J.W. Bode and E.M. Carreira, Tetrahedron Lett., 23, 4777 (1982); https://doi.org/10.1016/S0040-4039(00)85711-1.

10. V. Jager and H. Grund, Angew. Chem. Int. Ed. Engl., 15, 50 (1976); https://doi.org/10.1002/anie.197600501.

11. S.Y. Lee, B.S. Lee, C.W. Lee and D.Y. Oh, J. Org. Chem., 65, 256 (2000); https://doi.org/10.1021/jo991261y.

12. G.W. Moersch, E.L. Wittle and W.A. Neuklis, J. Org. Chem., 32, 1387 (1967); https://doi.org/10.1021/jo01280a023.
13. A. Yashiro, Y. Nishida, K. Kobayashi and M. Ohno, Synlett, 361 (2000); https://doi.org/10.1055/s-2000-6548.

14. P. Conti, C. Dallanoce, M. De Amici, C. De Micheli and K.-N. Klotz, Bioorg. Med. Chem., 6, 401 (1998); https://doi.org/10.1016/S0968-0896(97)10051-7.

15. A. Mishra, S.K. Jain and J.G. Asthana, Orient. J. Chem., 14, 151 (1998).

16. D.H. Ko, M.F. Maponya, M.A. Khalil, E.T. Oriaku and Z. You Lee, J. Med. Chem. Res., 8, 313 (1998).

17. Y.Y. Kang, K.J. Shin, K.H. Yoo, K.J. Seo, C.Y. Hong, C.S. Lee, S.Y. Park, D.J. Kim and S.W. Park, Bioorg. Med. Chem. Lett., 10, 95 (2000); https://doi.org/10.1016/S0960-894X(99)00646-0.

18. S. Srivastava, L.K. Bajpai, S. Batra, A.P. Bhaduri, J.P. Maikhuri, G. Gupta and J.D. Dhar, Bioorg. Med. Chem., 7, 2607 (1999); https://doi.org/10.1016/S0968-0896(99)00188-1.

19. P.A. Brough, W. Aherne, X. Barril, J. Borgognoni, K. Boxall, J.E. Cansfield, K.-M.J. Cheung, I. Collins, N.G.M. Davies, M.J. Drysdale, B. Dymock, S.A. Eccles, H. Finch, A. Fink, A. Hayes, R. Howes, R.E. Hubbard, K. James, A.M. Jordan, A. Lockie, V. Martins, A. Massey, T.P. Matthews, E. McDonald, C.J. Northfield, L.H. Pearl, C. Prodromou, S. Ray, F.I. Raynaud, S.D. Roughley, S.Y. Sharp, A. Surgenor, D.L. Walmsley, P. Webb, M. Wood, P. Workman and L. Wright, J. Med. Chem., 51, 196 (2008); https://doi.org/10.1021/jm701018h

20. A.G. Habeeb, P.N. Praveen Rao and E.E. Knaus, J. Med. Chem., 44, 2921 (2001) https://doi.org/10.1021/jm0101287.

21. B.M. Kwon, K.H. Son, D.C. Han, S.K. Lee, K.D. Shin, S.B. Jeon and J.H. Oh, US Patent 0131036 (2005)

22. R. Kamal, D. Sharma, D. Wadhwa and O. Prakash, Synlett., 93 (2012); https://doi.org/10.1055/s-0031-1290109.

23. L. Rebrovic and G.F. Koser, J. Org. Chem., 49, 2462 (1984); https://doi.org/10.1021/jo00187a032. 